EXPERIMENTAL CONSIDERATIONS CONCERNING THE VELOCITY MEASUREMENT OF THE RELATIVISTIC ELECTRON BEAM IN A GYROTRON BY MEANS OF THOMSON SCATTERING

M.R. Siegrist, G. Soumagne, M.Q. Tran

submitted for publication in Journal of Applied Physics 


\title{
Experimental considerations concerning the velocity measurement of the relativistic electron beam in a gyrotron by means of Thomson scattering
}

\author{
M. R. Siegrist, G. Soumagne, M. Q. Tran \\ Centre de Recherches en Physique des Plasmas, \\ Association Euratom - Confédération Suisse, \\ Ecole Polytechnique Fédérale de Lausanne, \\ 21, Avenue des Bains, 1007 Lausanne, Switzerland
}

\begin{abstract}
The feasibility of Thomson scattering to determine the beam velocity in a gyrotron has been analyzed and preliminary experiments to implement such a system on our $100 \mathrm{GHz}$ quasi-optical gyrotron are reported. Although the project had to be abandoned due to technical problems, the conclusions are that for the $90^{\circ}$ scattering arrangement discussed it should be possible to determine at least one velocity component with an acceptable signal-to-noise ratio.
\end{abstract}




\title{
Experimental considerations concerning the velocity measurement of the relativistic electron beam in a gyrotron by means of Thomson scattering
}

\author{
M. R. Siegrist, G. Soumagne, M. Q. Tran \\ Centre de Recherches en Physique des Plasmas, \\ Association Euratom - Confédération Suisse, \\ Ecole Polytechnique Fédérale de Lausanne, \\ 21, Avenue des Bains, 1007 Lausanne, Switzerland
}

\section{Introduction}

In gyrotrons the rotational energy contained in the relativistic electron beam is transferred to the electromagnetic radiation in the microwave cavity. The efficiency of this process is limited and usually significantly less than predicted. ${ }^{1}$ This is probably mainly due to poor beam quality and it is hoped that considerable improvements might be possible if the problems could be clearly identified.

In order to do that a precise beam diagnostic method has to be developed. Thomson scattering offers an accurate and non-intrusive technique which has been applied with great success in plasma physics. While the cross-section for Thomson scattering is very small, the scattered signal, which is proportional to the particle density, can easily reach a detectable level in tokamak-type plasmas, but the electron density in a gyrotron is one to two orders of magnitude smaller $\left(\approx 3 \cdot 10^{11} \mathrm{~cm}^{-3}\right)$. Hence considerable thought has to be given to the geometry of the interaction region and the light collection capability of the detection optics. ${ }^{2}$

In this paper we propose an arrangement which allows simultaneous measurement of the parallel and perpendicular beam velocity with an acceptable signal-to-noise ratio. Preliminary experiments are reported to implement this system on the quasi-optical $100 \mathrm{GHz}$ gyrotron of our institute. Unfortunately due to the very restrictive access to the interaction region and the scanty availability of the gyrotron for this project, the plans to build the system had to be abandoned. 


\section{Measurement of the electron beam velocity}

The electron beam in a gyrotron has two characteristic properties which make measurements with conventional methods relying on beam interception or capacitive coupling rather difficult: its high power density $\left(\geq 10 \mathrm{MW} / \mathrm{cm}^{2}\right)$ which causes rapid melting of most materials and the fact that most of the energy is contained in rotational motion. ${ }^{3-4}$ With these methods only the translational velocity component is measured and the perpendicular velocity, which actually couples to the RF- field of the resonator, is determined under the assumption of energy conservation which might be modified by the presence of parasitic modes in the electron beam duct.

Thomson scattering is a non-intrusive method and in principle offers great flexibility by a suitable choice of the scattering geometry which is, however, usually severely restricted by access.

Disregarding for the moment the fact that apart from access the particle density also limits the available choices of scattering arrangements, the relativistic velocity of the electrons offers a great advantage with respect to a fusion-type plasma: under most conditions the frequency of a scattered signal is shifted quite significantly from the original laser frequency. Hence parasitic stray light, one of the major noise sources in Thomson scattering from plasmas, can easily be suppressed with a suitable bandpass filter. We will first discuss qualitatively how it is possible to enhance the influence of a particular parameter on the spectrum with the scattering geometry, then derive the relevant formula and discuss calculated spectra. After this an estimation of the magnitude of the scattered signal and the signal-to-noise ratio will be given. In the final section experimental considerations concerning our $100 \mathrm{GHz}$ gyrotron will be given and the reason behind our decision to abandon the project at a relatively early stage will be outlined.

\section{Scattering geometry}

For an arbitrary scattering geometry the conditions are illustrated in Fig. 1 which shows the particle motion $\vec{v}$, the wave vector $\vec{k}_{0}$ of the incident and $\vec{k}_{s}$ of the scattered radiation. Each electron of the gyrotron e-beam describes a helical motion around the 
magnetic field lines which are parallel to the symmetry axis of the gyrotron in the interaction region. Hence their velocity distribution can be described by a parallel and a perpendicular velocity component and their respective velocity spreads. The angle of the velocity vector with the symmetry axis is referred to as pitch angle $\Phi$.

The frequency of the scattered radiation $\omega_{s}$ is related to the frequency $\omega_{0}$ of the incident wave by the Doppler formula ${ }^{5}$

$$
\omega_{s}=\omega_{0} \frac{1-\beta \cos \theta}{1-\beta \cos \theta^{\prime}}
$$

where $\beta$ is the beam velocity in units of the velocity of light $(\beta=v / c)$, and $\theta, \theta^{\prime}$ are the angles between the velocity vector $\vec{v}$ and the incident and scattered wave vectors $\vec{k}_{0}$ and $\vec{k}_{s}$, respectively.

The frequency ratio $\omega_{s} / \omega_{0}$ depends therefore on $\beta$ and on the scattering geometry given by $\vec{k}_{0}$ and $\vec{k}_{s}$, which allows us to adjust the frequency shift in order to match the spectral response curve of the detector within certain limits. For a defined geometry the observed frequency change depends only on the velocity vector of the scattering particles. To illustrate this dependence we plot in Figs. 2,3,4 the ratio $\omega_{s} / \omega_{0}$ as a function of $\theta$ for a constant absolute value of $\beta$ (monoenergetic e-beam) and fixed directions of the vectors $\vec{k}_{0}$ and $\vec{k}_{s}$. These two vectors define the scattering plane and the angle between them is known as the scattering angle $\gamma$. To simplify the discussion we will in the following assume that $\vec{v}$ is in the scattering plane.

From standard non-relativistic scattering theory ${ }^{6}$ it is well known that the differential scattering vector $\vec{k}_{d}=\vec{k}_{0}-\vec{k}_{s}$ indicates the direction in which the velocity distribution is sampled. This can directly be seen for different geometries $\left(\gamma=1^{\circ}, 90^{\circ}, 180^{\circ}\right)$ and a low $\beta$-value $(\beta=0.0047)$ in Fig. 2a, 3a, 4a. The up- and downshift in frequency is very small $\left(\omega_{s} \approx \omega_{0}\right)$, symmetric and has its maximum value for velocity vectors parallel and anti parallel to $\vec{k}_{d}$. In particular, when the velocity vector is parallel (anti parallel) to $\vec{k}_{d}$ the relative frequency shift is negative (positive). Note that for $\gamma=1^{\circ}$ (forward scattering) the frequency shift is much smaller than for $\gamma=180^{\circ}$ (back scattering), that there is no shift for $\gamma=0^{\circ}$ and that no shift occurs for velocities perpendicular to $\vec{k}_{d}$. The relative frequency shift for $\beta \ll 1$ is given by

$$
\frac{\Delta \omega}{\omega_{0}}=\frac{\omega_{s}-\omega_{0}}{\omega_{0}}=-\frac{2 v_{p}}{c} \sin \frac{\gamma}{2}
$$


where $\mathrm{c}$ is the vacuum velocity of light and $v_{p}$ the projection of $\vec{v}$ onto $\vec{k}_{d}$.

In the case of relativistic velocities the dependence changes, as can be seen in Fig. $2 \mathrm{~b}, 3 \mathrm{~b}, 4 \mathrm{~b}$ for $\beta=0.47$ which corresponds to a typical value of the electron beam in our gyrotron. First, note that in general the shift is much larger than in the classical case and that it is normally no longer symmetric with respect to the incident frequency. Second, the differential scattering vector is no longer fixed in space because the frequency shift is now so large that the length of $\vec{k}_{s}$ changes significantly. As a consequence the velocity vectors which produce the maximum up- and downshifts are, except for back scattering, no longer anti parallel to each other. The spectrum is therefore not determined by a single velocity component and the simple relationship of Eq. (3.2) is no longer valid. In addition, at different absolute $\beta$-values the maximum up- and downshift will occur at different angles of the velocity vector, but this effect is negligible in our case as can be seen in Fig. 5 . Hence, in general, a single measurement at one particular scattering geometry will not provide enough information to determine the velocity distribution of a given component. ${ }^{7}$ As we will see later it is however possible in our application to determine the mean velocity vector from a single spectrum due to a restriction of the $\theta$-values by certain geometries. Third, the direction of $\beta$ which does not produce any frequency shift at all is still the bisecting line of $\vec{k}_{0}$ and $\vec{k}_{s}$. This follows directly from the Doppler formula, Eq. (3.1) which reduces to $\omega_{s}=\omega_{0}$ for $\theta=\theta^{\prime}$. Therefore the angle $\psi$, which is the angle between the velocity vectors which produce the maximum shift (up or down) and no shift at all, changes as well. The angle is given by

$$
\cos \psi=\beta \cos \frac{\gamma}{2}
$$

where $\gamma$ is the scattering angle. As long as $\beta$ is not too large, $\psi$ is close to $90^{\circ}$, and this is true even for quite large $\beta$ in the case of back-scattering $\left(\gamma=180^{\circ}\right)$. For forward scattering $\left(\gamma=1^{\circ}, \beta=0.47\right)$ the maximum shift towards higher and lower frequencies now occurs at an angle $\psi= \pm 62^{\circ}$ according to equation 3.3. Since $\theta=\psi+\gamma / 2$ the maximum shift to higher and lower frequencies occurs at $62.5^{\circ}$ and $298.5^{\circ}$, which means that the corresponding velocity vectors form an angle of $124^{\circ}$ instead of $180^{\circ}$ as in the classical case. In this case the shift is still symmetric with respect to the incident frequency, similar to the non-relativistic case. The vector diagram in Fig. 2 shows that a change in the 
length of $\vec{k}_{s}$ due to a change of $\beta$ rotates the vector $\vec{k}_{d}$ by a significant amount. Hence the largest frequency shift for each $\beta$ is obtained for a different direction of the velocity vector and it is unlikely that the two effects (length and angle dependence) are separable.

In the case of back scattering $\vec{k}_{d}$ is fixed (parallel to $\vec{k}_{0}$ ) and the velocity component $v_{\|}$can be measured, but all information about the pitch angle $\Phi$ is lost.

For $\gamma=90^{\circ}$ both $\beta$ and the velocity direction influence the spectrum. The angle of $\vec{k}_{d}$ defining the direction which produces a maximum frequency shift, is variable. This raises hopes that it might be possible to obtain information on both $\beta$ and the direction of the velocity vector from the spectrum, provided the two effects are not too scrambled. We will indeed find later on that this is the case. For our case $\psi=70.6^{\circ}$ and the two angles for maximum shift are $\theta_{\max }=115.6^{\circ}, 334.4^{\circ}$.

In the following we will discuss different scattering arrangements, whereby we assume that $\beta \ll 1$, for simplicity. The vector diagrams are shown in Fig. 2, 3, 4 .

\subsection{Collinear arrangements}

With the term collinear arrangements we mean that both the incident and scattered radiation vectors are parallel, or at least almost parallel to the gyrotron axis of symmetry. Notwithstanding mechanical constraints (the presence of the gun) we can consider forward and backward scattering parallel or anti parallel to the electron beam.

For backward scattering the differential scattering vector is parallel to $v_{\|}$for any $\beta$. Hence $v_{\perp}$ does not influence the spectrum and the parallel velocity distribution can be measured. The relative frequency shift according to Eq. (3.1) is maximum and for headon electron-photon collisions $\theta=180^{\circ}$ given by:

$$
\Delta \omega / \omega_{0}=2 \beta_{\|} /\left(1-\beta_{\|}\right)
$$

This reduces to $2 \beta$ for $\beta \ll 1$ according to Eq. (3.2). (Note that $\vec{v}_{p}$ is anti parallel to $\vec{k}_{d}$ in this case and hence negative, so that $\Delta \omega$ in Eq. (3.2) is positive).

If the incident radiation propagates in the same direction as the electrons the same velocity component is measured, but the frequency shifts in the opposite direction with the ratio $\omega_{s} / \omega_{0}$ being the inverse of the previous case. 
For exact forward scattering $\left(\theta=\theta^{\prime}=0\right)$ no shift is obtained, but for small angle forward scattering the differential scattering vector is perpendicular to the beam axis and hence allows to measure $v_{\perp}$, at least in the classical case, whereas a mixing of the two components occurs at relativistic velocities. The frequency shift is small, but if the incident narrow-band laser radiation can be suppressed efficiently enough with a narrow-band filter, this might still be an interesting arrangement.

It should be remembered that the electrons describe a circular motion in the plane perpendicular to the beam axis. This means that the velocity components of individual electrons projected on the scattering plane take all values between $-v_{\perp}$ and $+v_{\perp}$ and hence also the frequency shift according to Eq. (3.2) covers a corresponding range. It is to be expected that a spectrum enlarged in this way completely masks the much smaller effect of a velocity spread.

Note that in all collinear arrangements the scattering volume is rather elongated which enhances the scattering intensity, but is unfavorable for spatial resolution. The incoming or scattered radiation either hits the cathode or passes very close to it which may not be possible due to access restrictions.

\subsection{Forward and backward scattering perpendicular to the beam axis}

Here again we have the possibility to observe either $v_{\|}$(near forward scattering) or $v_{\perp}$ (backward scattering). The frequency shift for near forward scattering is small, but it may again be possible to suppress the unshifted laser radiation with filters. Backward scattering produces a broad spectrum, filling out the region corresponding to shifts caused by $-v_{\perp}$ and $+v_{\perp}$.

Although good spatial resolution could be obtained, the scattering volume and hence the scattering intensity are tiny in these arrangements which, therefore, do not deserve further discussion. 


\subsection{Right-angle scattering}

Of all the possible right-angle scattering arrangements only those with the incident radiation parallel or anti parallel to the gyrotron axis are interesting. Indeed, the cylindrical symmetry of the system allows to enhance considerably the amount of collected radiation, as will be shown further on.

The differential scattering vector for $\beta \ll 1$ forms an angle of $45^{\circ}$ with the axis and hence both $v_{\|}$and $v_{\perp}$ are sampled. When the incident radiation is propagating in the opposite direction to the electron beam, described by $\beta=0.47$ and $\Phi=55^{\circ}$ (pitch angle), the angle $\theta$ is fixed at $125^{\circ}$. The expected maximum up- and downshift is therefore 2.1 and 0.9 times the incident frequency, corresponding to $\theta^{\prime}=35^{\circ}$ and $\theta^{\prime}=145^{\circ}$ respectively (Fig. $3 b)$. Furthermore, for velocity vectors which are not in the scattering plane the $\theta^{\prime}$-values are in the range of $35^{\circ}$ to $145^{\circ}$ and the shift they produce will be smaller according to Eq. (3.1). The spectrum therefore covers the whole spectral range between the maximum shifts. For $\Phi=55^{\circ}$ the high frequency end of the spectrum depends less on a change of the pitch angle than the low frequency end as can be seen by the smaller slope at this point (Fig. 3b).

The opposite holds for a change in velocity as can be seen in Fig. 5 . While our qualitative discussion is insufficient to predict the form of the spectrum, it can be assumed that such an arrangement allows to measure simultaneously the pitch angle and $\beta$ and hence $v_{\|}$and $v_{\perp}$. The gyro motion will, of course, smear out the $v_{\perp}$ contribution again, since velocity components in the range $-v_{\perp}$ to $+v_{\perp}$ all contribute to the signal. Note that a higher $\beta$ with lower pitch angle can yield the same maximum high frequency shift as a lower $\beta$ with higher pitch angle. In order to determine the pitch angle and $\beta$ one therefore has to observe the low frequency end of the spectrum as well. As will be shown later this is not feasible in our case. An additional diagnostic would be required to measure both the pitch angle and $\beta$.

A numerical code has been developed to discuss the resulting spectra in more detail. It will be presented in the following section. 


\section{Thomson scattering from a weakly relativistic electron beam}

A detailed theoretical study of the spectrum of electromagnetic radiation scattered by a strongly relativistic electron beam has been reported by Zhuravlev et al. ${ }^{8}$ In particular, they investigated the influence of the scattering geometry, of the relativistic particle velocities and the polarization characteristics of the radiation. While they demonstrated that interesting supplementary information can be obtained from polarization measurements, ${ }^{9}$ we will not treat these effects in this paper, although the required correction terms could easily be incorporated into our numerical code. Instead we will try to illustrate, what kind of information can be obtained in an actual measurement and which are the important parameters to adjust.

We start from Eq. 8 of Ref. 8 for the scattering form factor S, adapted to the notation used in this paper

$$
S\left(\vec{k}_{d}, \omega\right)=\iiint d^{3} v r(\vec{v}) f(\vec{v}) \delta\left(\omega-\frac{v \cos \theta^{\prime}-v \cos \theta}{1-v \cos \theta^{\prime}}\right)
$$

where $\omega$ now stands for the dimensionless frequency shift $\Delta \omega / \omega_{0}$. The relativistic correction term $r(\vec{v})$ will not be further discussed here, but is included in the code for the case of the polarization of the incident and scattered light perpendicular to the scattering plane. The $v \cos \theta$-term can be replaced by the scalar products $\vec{e} \cdot \vec{v}$ and using $\vec{a}=\vec{e}_{0}-\vec{e}_{s}$ where $\vec{e}_{0}, \vec{e}_{s}$ are unit vectors parallel to $\vec{k}_{0}, \vec{k}_{s}$, we find

$$
\begin{aligned}
S\left(\vec{k}_{d}, \omega\right) & =\iiint d v_{x} d v_{y} d v_{z} r(\vec{v}) f(\vec{v}) \delta\left(\omega+\frac{\vec{v} \cdot \vec{a}}{1-\vec{v} \cdot e_{s}}\right) \\
& =\iiint d v_{x} d v_{y} d v_{z} r(\vec{v}) f(\vec{v}) \delta\left(\omega+\frac{v_{x} a_{x}+v_{y} a_{y}+v_{z} a_{z}}{1-v_{x} e_{s x}-v_{y} e_{s y}-v_{z} e_{s z}}\right)
\end{aligned}
$$

After integration over $v_{z}$ we obtain:

$$
\begin{aligned}
S\left(\vec{k}_{d}, \omega\right) & =-\iint d v_{x} d v_{y} f\left(v_{x}, v_{y}, v_{z}=\frac{\omega\left(1-v_{x} e_{s x}-v_{y} e_{s y}\right)+v_{x} a_{x}+v_{y} a_{y}}{\omega e_{s z}-e_{z}}\right) \\
& \cdot r\left(v_{x}, v_{y}, v_{z}=\frac{\omega\left(1-v_{x} e_{s x}-v_{y} e_{s y}\right)+v_{x} a_{x}+v_{y} a_{y}}{\omega e_{s z}-e_{z}}\right) \\
& \cdot \frac{e_{s z}\left(v_{x} a_{x}+v_{y} a_{y}\right)+e_{z}\left(1-v_{x} e_{s x}-v_{y} e_{s y}\right)}{\left(\omega e_{s z}-e_{z}\right)^{2}}
\end{aligned}
$$

This is the equation which is numerically integrated in our code, whereby we assume that $f(\vec{v})$ is Gaussian with widths $\Delta v_{\|}$and $\Delta v_{\perp}$. (Note that $\vec{a}=\vec{k}_{d} /\left|\vec{k}_{0}\right|$ in the non-relativistic 
case. It has otherwise no particular meaning except that it depends only on the scattering geometry in a simple way).

\section{Discussion of spectra for the case of right-angle scattering}

We used the numerical code based on Eq. (4.3) to predict spectra for the right-angle scattering arrangement discussed in section 3.3. We assume a Nd:glass laser at $1.06 \mu m$ wavelength as laser source and beam parameters which are typical for our $100 \mathrm{GHz}$ gyrotron. Fig. 6a shows spectra for 3 different pitch angles $\phi$ (angle between beam velocity vector and axis of symmetry). As can be seen, changing the pitch angle influences the low-frequency peak in the vicinity of the laser line. A clearly measurable displacement towards higher wavelength is observed as the pitch angle is increased. While at $\phi=50^{\circ}$ the peak still overlaps with the unshifted laser line, filtering out the latter one at $\phi \geq 55^{\circ}$ seems feasible. Unfortunately this takes place in a spectral region where sensitive detectors are not readily available.

While the high frequency peak is practically unaffected by the pitch angle, it is rather sensitive to the absolute electron velocity or to $\beta$. This is shown in Fig. $6 \mathrm{~b}$ where a $10 \%$ variation in $\beta$ shifts the position of the peak by about one peak width.

Thus in principle this scattering geometry allows us to measure both pitch angle and $\beta$ and hence both components of the velocity vector: $v_{\|}$and $v_{\perp}$. The attainable precision depends to a large degree on the number of spectral channels available and hence on photon numbers. This point will be discussed in section 6 .

In Figs. 7a, 7b we show the spectra which correspond to the parameters of Figs. 6a, $6 \mathrm{~b}$, except that a ruby laser is used as source. The low frequency peak is now in a range where photo multipliers with good sensitivity are available, but the peak which reveals the $\beta$-information is in the UV. Ideally a combination of $\mathrm{Nd}$ :glass and ruby laser would yield an optimum amount of information, but in practice such a system would be too complex and expensive.

Although it seems unlikely to be able to determine the velocity spread in a realistic set-up, we show nevertheless in Fig. 8 the effect of a variation of the width of the velocity distribution, assumed to be gaussian. Changing $\Delta v_{\|}$or $\Delta v_{\perp}$ neither results in a significant 
shift nor broadening of the peak. Only the height of the peak is affected which is difficult to measure and requires a careful intensity calibration. Hence this arrangement is not suitable to measure velocity spreads.

\section{Estimation of count rates}

In view of the small cross-section for Thomson scattering and the low beam density, detection of the scattered radiation will be difficult and only the most sensitive detection system will be adequate. Our discussion is based on the use of a photo multiplier combined with a monochromator or a set of optical bandpath filters. A spectrum cannot be obtained in a single shot, but must be built up over a series of shots.

The ratio of scattered intensity to incident intensity according to standard theory of Thomson scattering is

$$
I_{s} / I_{0}=\sigma_{T} \cdot n \cdot \Delta \Omega \cdot l \cdot \int S(\omega) d \omega
$$

with

$I_{s} / I_{0}$ ratio of scattered intensity

$\sigma_{T}$ Thomson cross-section

$n$ electron density

$\Delta \Omega$ solid angle

$l$ length of scattering region

$S(\omega)$ scattering form factor

The integrated form factor in the non-collective regime can be set to one. The electron density in our case is typically $3 \cdot 10^{11} \mathrm{~cm}^{-3}$ and we assume that an overlap region between the focused laser radiation and the electron beam of $5 \mathrm{~cm}$ length can be imaged onto the detector. For the estimation of the solid angle we assume that with the use of a cylindrical mirror of parabolic shape a collection angle of $90^{\circ}$ can be sustained in the plane perpendicular to the beam and $10^{\circ}$ in the plane of the beam (see Fig. 9). This results in a solid angle of $\Delta \Omega=0.02 / 4 \pi s r$.

With $\sigma_{T}=6.65 \cdot 10^{-25} \mathrm{~cm}^{-2}$ the intensity ratio is obtained as $1.6 \cdot 10^{-15}$. The energy of a photon of wavelength $1 \mu \mathrm{m}$ is $2 \cdot 10^{-19} \mathrm{~J}$, so that 8000 photons are collected from a laser pulse of $1 \mathrm{~J}$. With a quantum efficiency of the photo multiplier of $20 \%$ and assuming that 
the total spectral width is divided into 10 channels, 160 photoelectrons are obtained on the average per channel, with statistical fluctuations - and hence error - below $10 \%$. The main sources of stray light are the unshifted laser radiation, which can be suppressed efficiently by a suitable notch filter, and the emission of the cathode which is heated to about $900^{\circ} \mathrm{C}$. With the proposed scattering geometry neither of these two noise sources are seen directly by the detection system. Only radiation which has been scattered several times from the metallic surfaces could find its way to the detector. Although theoretical estimates are unreliable, they indicate that neither of these noise sources should be a problem. Hence according to this the measurement just seems to be feasible, at least if optical transmission losses which have not been included above are not too important.

Lasers are available which produce considerably higher output energies than the $1 \mathrm{~J}$ assumed. The problem with the chosen scattering geometry is, that the laser beam strikes the cathode of the gyrotron. The careful shaping of the emitted electrons into a spiraling beam of low velocity spread should by no means be interfered with by laser induced photo emission. This problem is discussed in the next section.

\section{Breakdown}

The only geometry which allows us to collect a sufficient number of scattered photons is $90^{\circ}$ scattering with the incident laser beam anti parallel to the electron beam. The laser beam hits the electron gun in this case and may well impair its correct functioning. In order to investigate this constraint we installed a Nd:glass laser at the collector side of the gyrotron. With a distance between the interaction region and the collector of about $4 \mathrm{~m}$, the last focusing element (lens) of the laser beam is almost at a distance of $5 \mathrm{~m}$ from the scattering volume. In addition, the diameter is limited by the entrance window $(10 \mathrm{~cm})$ and by the beam duct inside the gyrotron vacuum vessel (Fig. 10). In order to focus $1 \mathrm{~J}$ into the scattering volume with its cross-section of $0.2 \mathrm{~mm}^{2}$, given by the annular electron beam, we needed about 5J. Without magnetic field we applied $30 \mathrm{kV}$ between the cathode and the first anode (three electrode gun, see Fig. 11) in order to simulate the electric field which accelerates the electrons during gyrotron operation. 
By firing $5 \mathrm{~J}$ of laser energy (pulse length $\approx 300 \mu s$ ) onto the heated gun arcs have been observed by a voltage drop of the $30 \mathrm{kV}$ low current power supply as well as by the short circuit current monitored by a Rogowsky coil. A pressure increase occurred at the same time. The front region of the gun (Fig. 11), the main component hit by the laser, is made of molybdenum and the incident average energy density of $\approx 5 \mathrm{~J} / \mathrm{cm}^{2}$ is well below the reported melting threshold of different molybdenum surfaces at $2.7 \mu m$ and $100 \mathrm{~ns}{ }^{10}$ Possible explanations for the arcs may be the high applied electric field $(\approx 10 \mathrm{kV} / \mathrm{mm})$ in correlation with the roughness of the surface (laser induced point discharge), laser evaporated impurities of the molybdenum, the 'spiking mode' of the laser and/or to the considerable percentage of the laser energy which hits the electron emitting cathode directly or after several reflections in the gun region. In situ cleaning of the molybdenum by the laser and by heating did not improve the situation. Mechanical constraints did not allow us to access the gun region and to investigate the interaction more quantitatively. We determined the threshold to be about 2-3J which corresponds to about 60-90 photoelectrons in the spectral channel with the highest signal.

At this stage we decided to abandon the experiment although the measurement may just have been feasible. The aim of the experiment was to determine the velocity distribution in the electron beam of our gyrotron and not a proof of principle of the Thomson scattering method which may well work under more favorable conditions (access). The development time and expenditure of the optical detection system, in particular the cylindrical collection mirror with parabolic surface, could not be justified and additional uncertainties had to be envisaged, as for example the presence of the magnetic field which will probably further decrease the threshold of the interaction. The reason is that the probability for ionization of evaporated electrons is increased by the longer path in the spiraling motion, in particular through interaction with the electron beam itself, a process which had already limited the gyrotron operation before laser application.

In conclusion, we have shown that a Thomson scattering diagnostic system to determine the velocity components of the e-beam in a gyrotron may be feasible if it is not impaired by too many additional constraints such as access to the interaction region. It would provide accurate measurements and could enhance considerably the understanding of the operation of gyrotrons and hence provide guidelines for improvement of performance. 
Unfortunately our own gyrotron imposed too many restrictions on the planned experiment which therefore had to be abandoned.

This work was partially supported by the Fond National Suisse pour la Recherche Scientifique and by the Office Fédérale de l'Energie under grant OFEN-Gyrotron 581-233.

\section{References}

1. S. Alberti, M.Q. Tran, J.P. Hogge, T.M. Tran, A. Bondeson, P. Muggli, A. Perrenoud, B. Joedicke, and H.G. Mathews, Phys. Fluids B $\underline{2}, 1654-1661$ (1990).

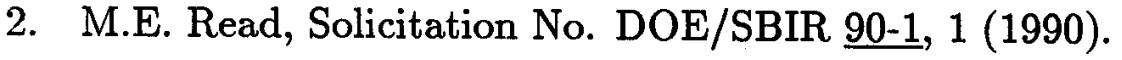

3. E.G. Avdoshin, L.V. Nikolaev, I.N. Platonov, and S.E. Tsimring, Radiophys. Quantum Elect. 16, 1241-1246 (1973).

4. W.C. Guss, T.L. Grimm, K.E. Kreischer, J.T. Polevoy, and R.J. Temkin, J. Appl. Phys. $\underline{69}$, 3789-3795 (1991).

5. C. Habfast, H. Poth, B. Seligmann, A. Wolf, J. Berger, P. Blatt, P. Hauck, W. Meyer, and R. Neumann, Appl. Phys. B 44, 87-92 (1987).

6. J. Sheffield, Plasma scattering of elecromagnetic radiation, Academic Press, 1977.

7. J.H. Williamson, and M.E. Clarke, J. Plasma Phys. $\underline{6}$, 211-221 (1971).

8. V.A. Zhuravlev, V.E. Muzalevskii, and G.D. Petrov, Sov. J. Plasma Phys. 7 , 292-295 (1981).

9. A.B. Kukushkin, and V.I. Kogan, Sov. J. Plasma Phys. $\underline{7}$, 669-672 (1981).

10. J.M. Bennett, P.C. Archibald, J.P. Rahn, and A. Klugman, Appl. Opt. 22, 4048-4055, (1981). 


\section{Figure captions}

Figure 1: The scattering geometry with the wave vectors and the electron motion. The velocity vectors form a cone with pitch angle $\Phi$.

Figure 2: The frequency shift as function of the angle $\theta$ between electron velocity and laser beam direction for near forward scattering.

Figure 3: As Fig. 2 for $90^{\circ}$-scattering.

Figure 4: As Fig. 2 for back-scattering.

Figure 5: As Fig. 3 for 3 different beam velocities.

Figure 6a: The wavelength spectrum for 3 different pitch angles.

Figure 6b: The wavelength spectrum for 3 different velocities.

Figure 7a: As Fig. 6a, but for a ruby laser.

Figure 7b: As Fig. 6b, but for a ruby laser.

Figure 8a: The wavelength spectrum for 3 different perpendicular velocity spreads.

Figure 8b: The wavelength spectrum for 3 different parallel velocity spreads.

Figure 9: The light collection in the interaction region by means of a parabolic mirror.

Figure 10: Cross-section of the gyrotron and the Thomson scattering system.

Figure 11: Cross-section of the 3 electode gun of our gyrotron. 

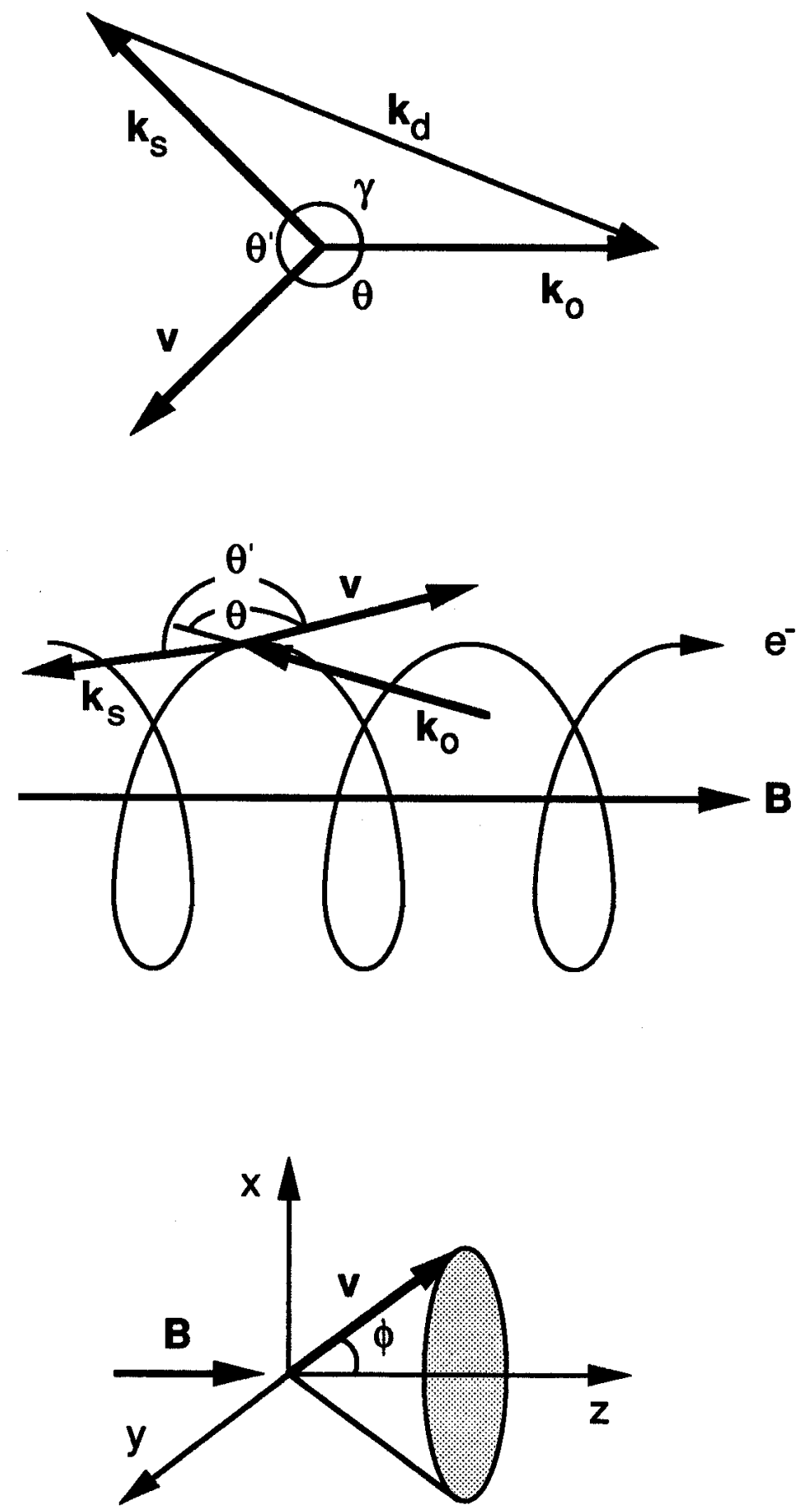

Fig, 1 


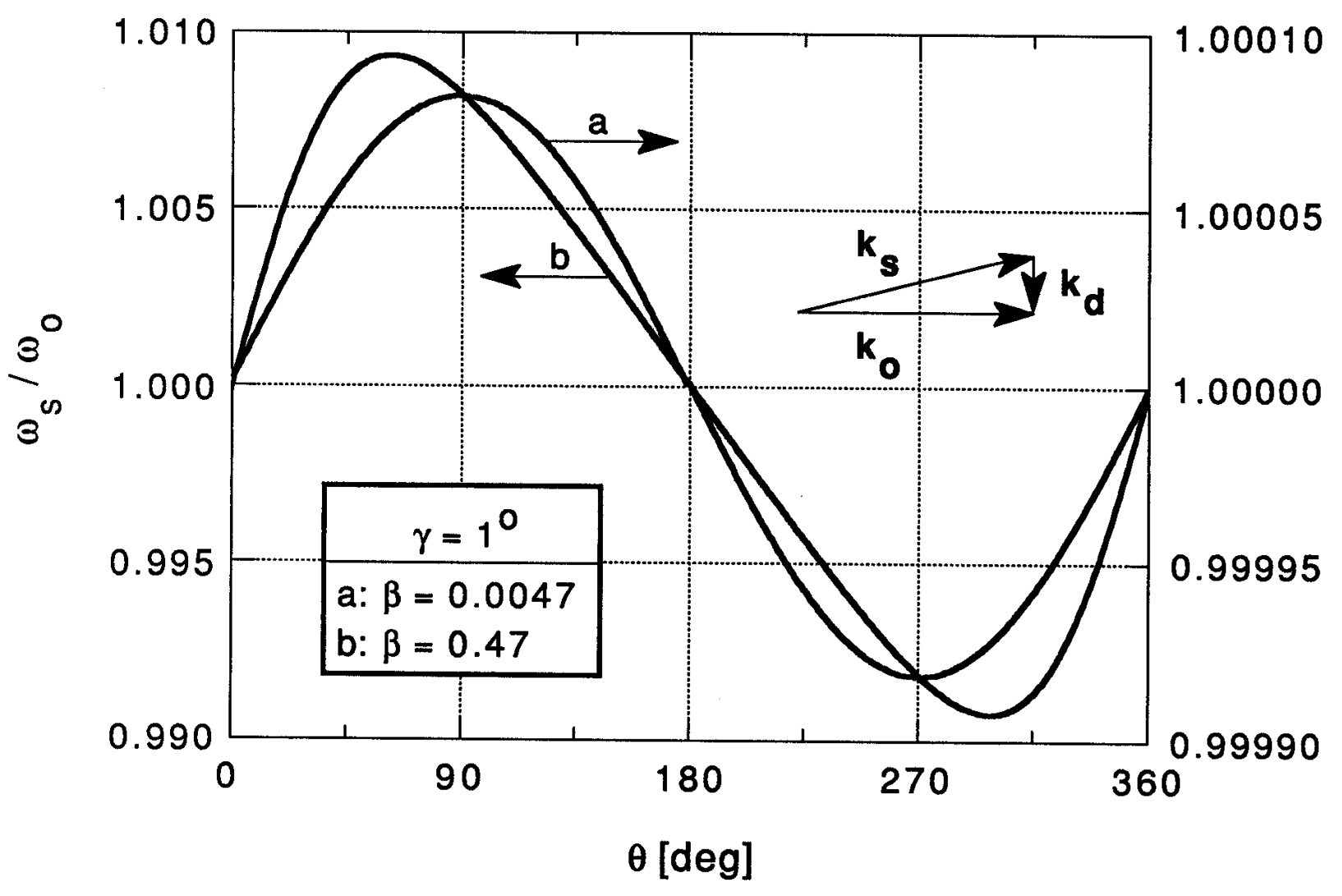

Fig. 2 


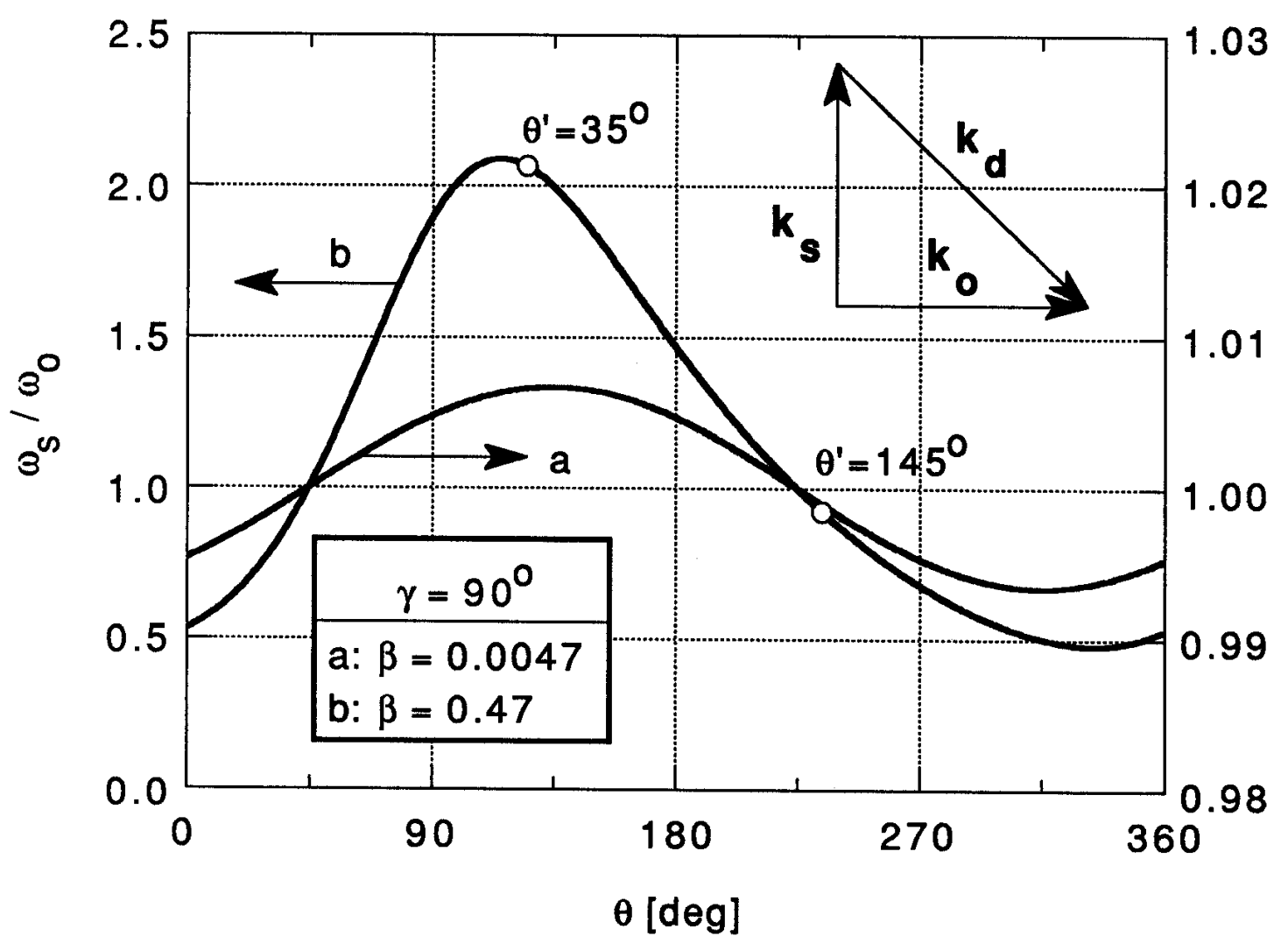

Fig. 3 


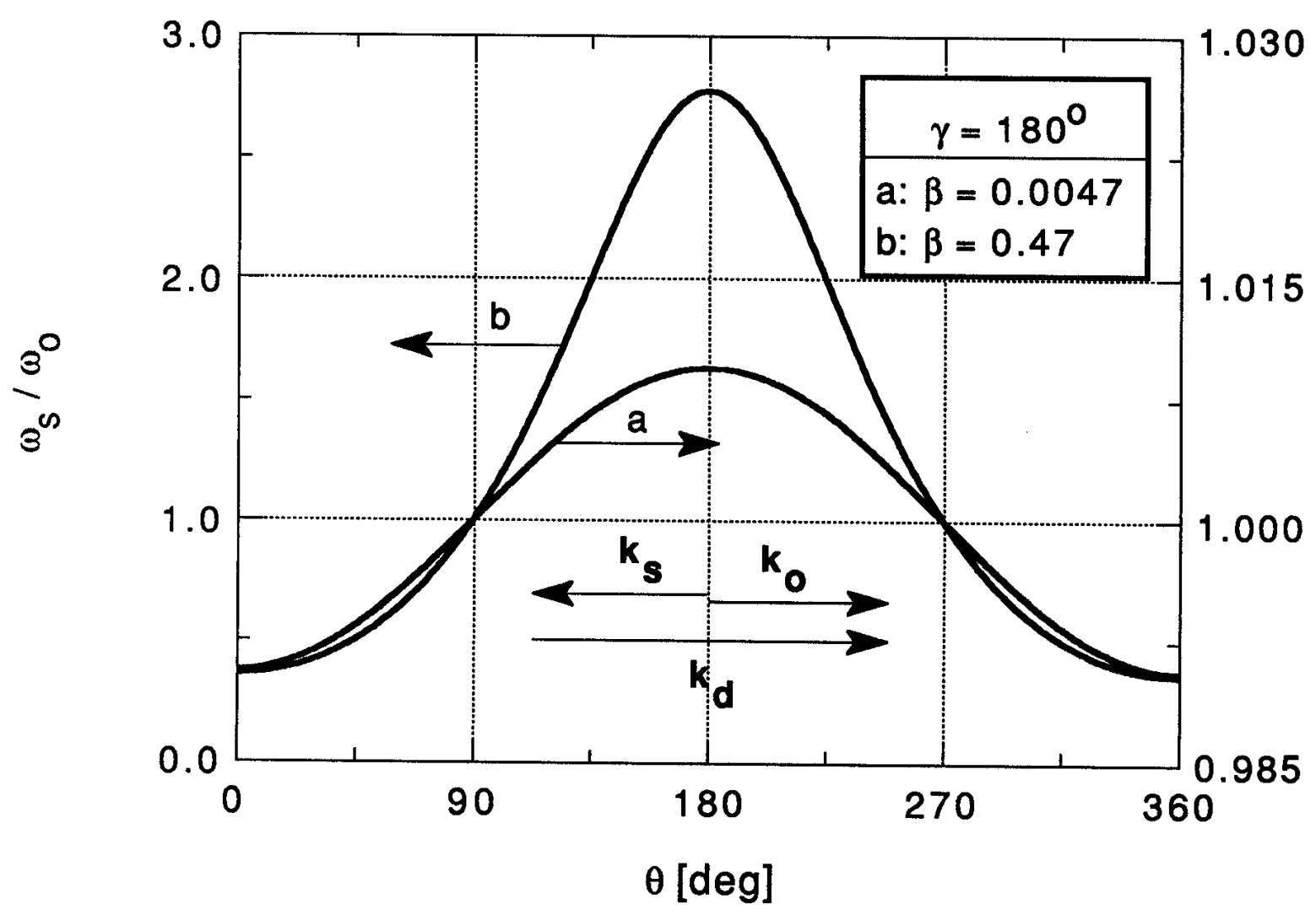

Fig. 4 


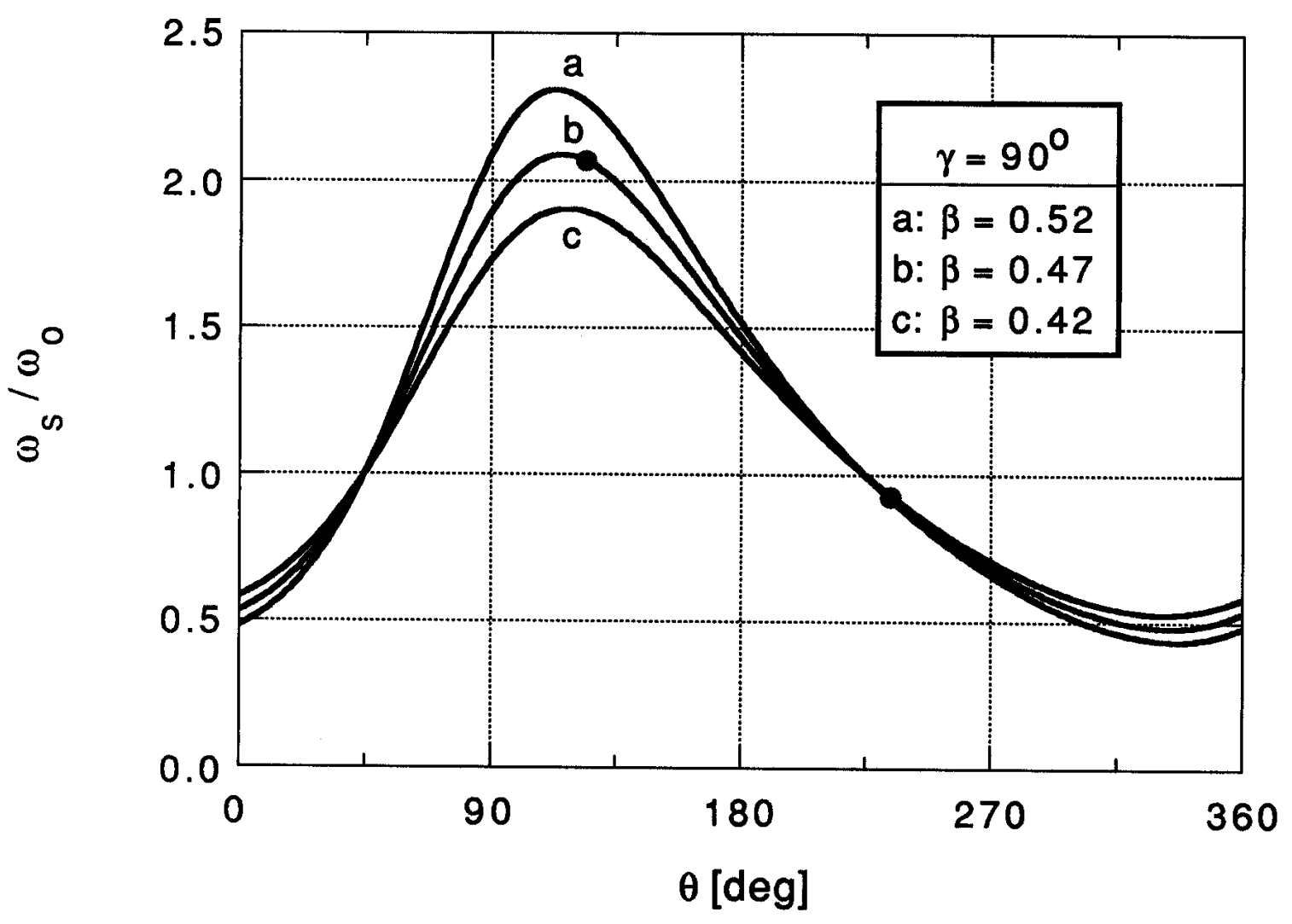

Fig, 5 


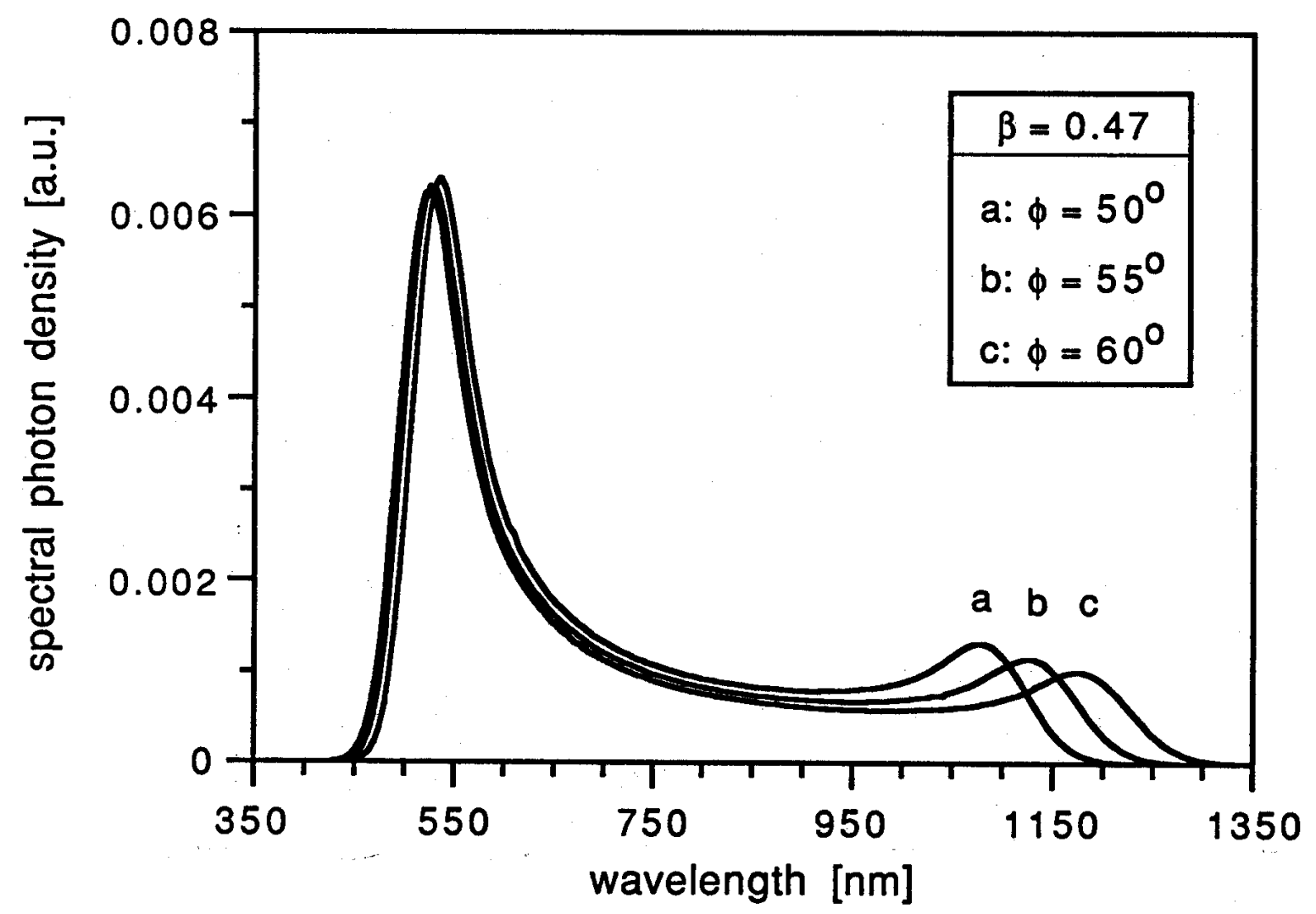

Fig, 6(a) 


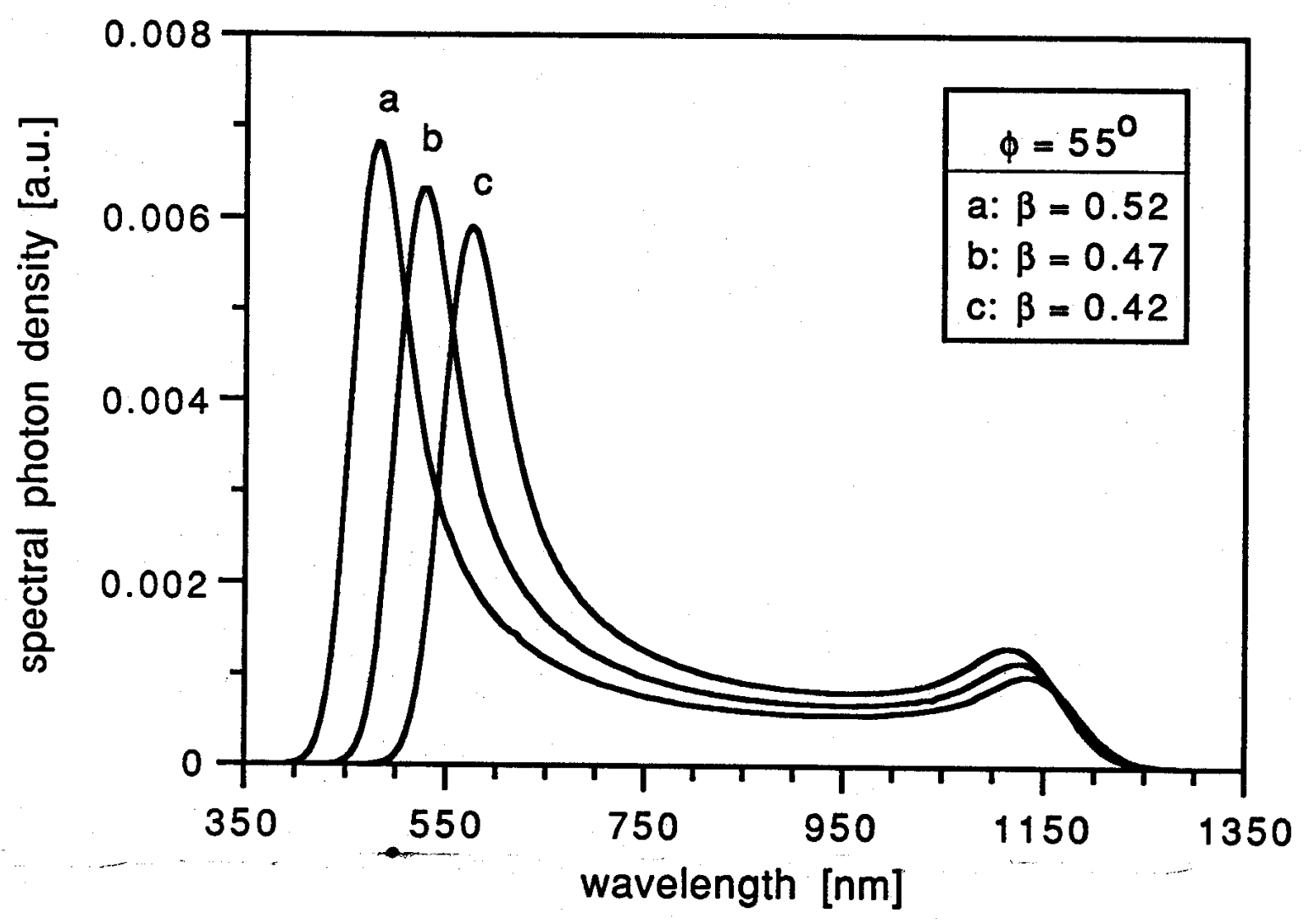

Fig, 6(b) 


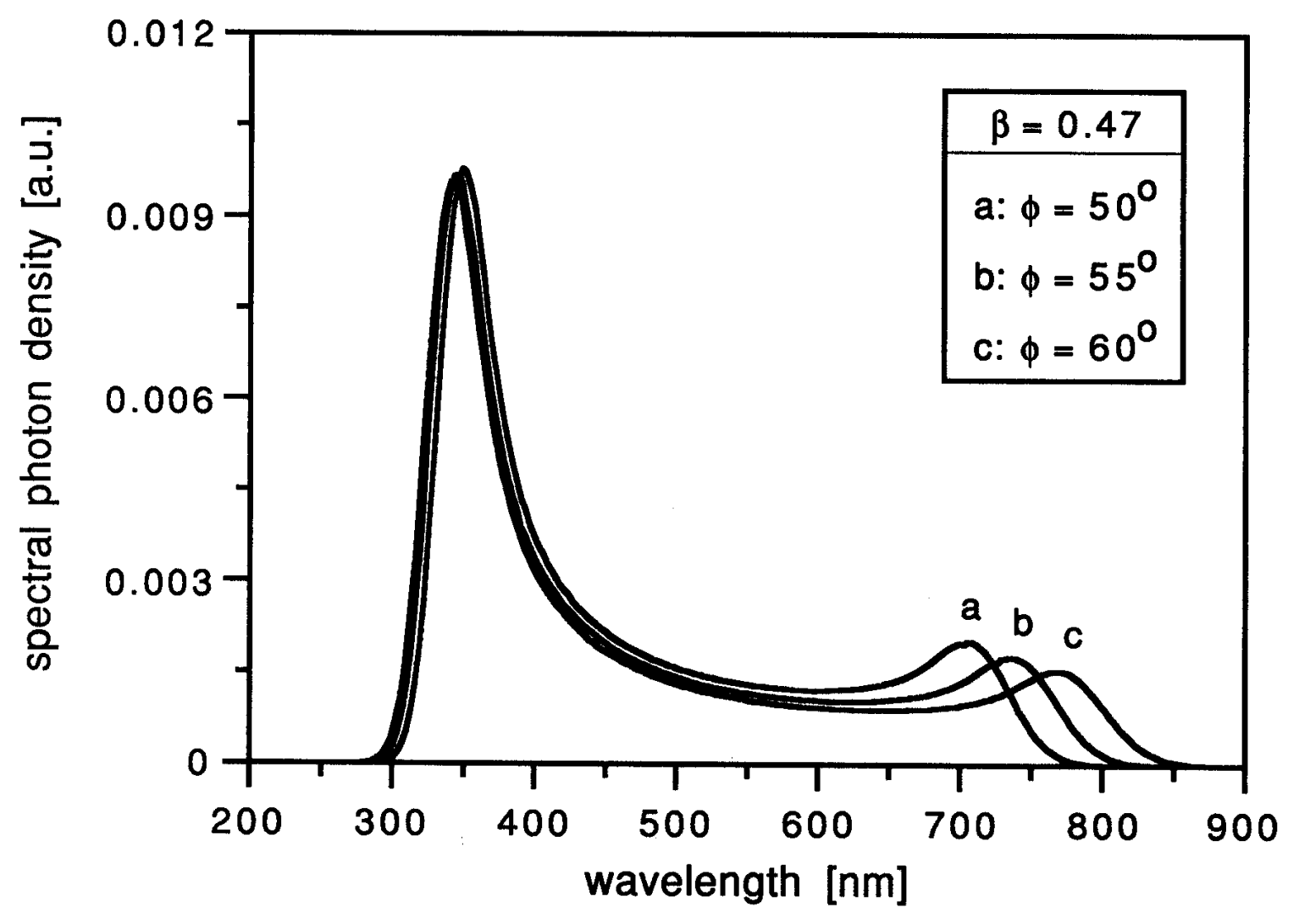

Fig, 7(a) 


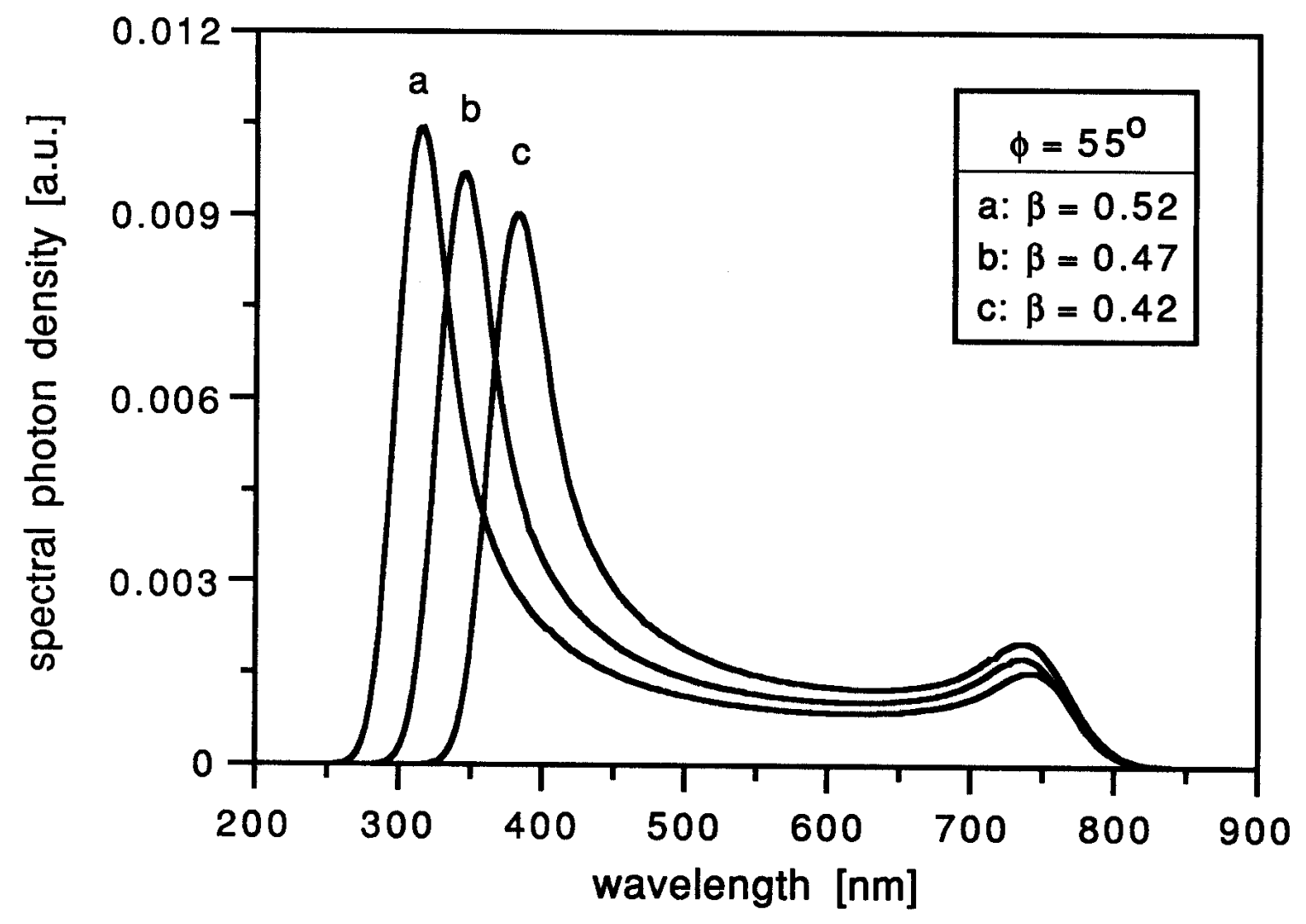

Fig, 7(b) 


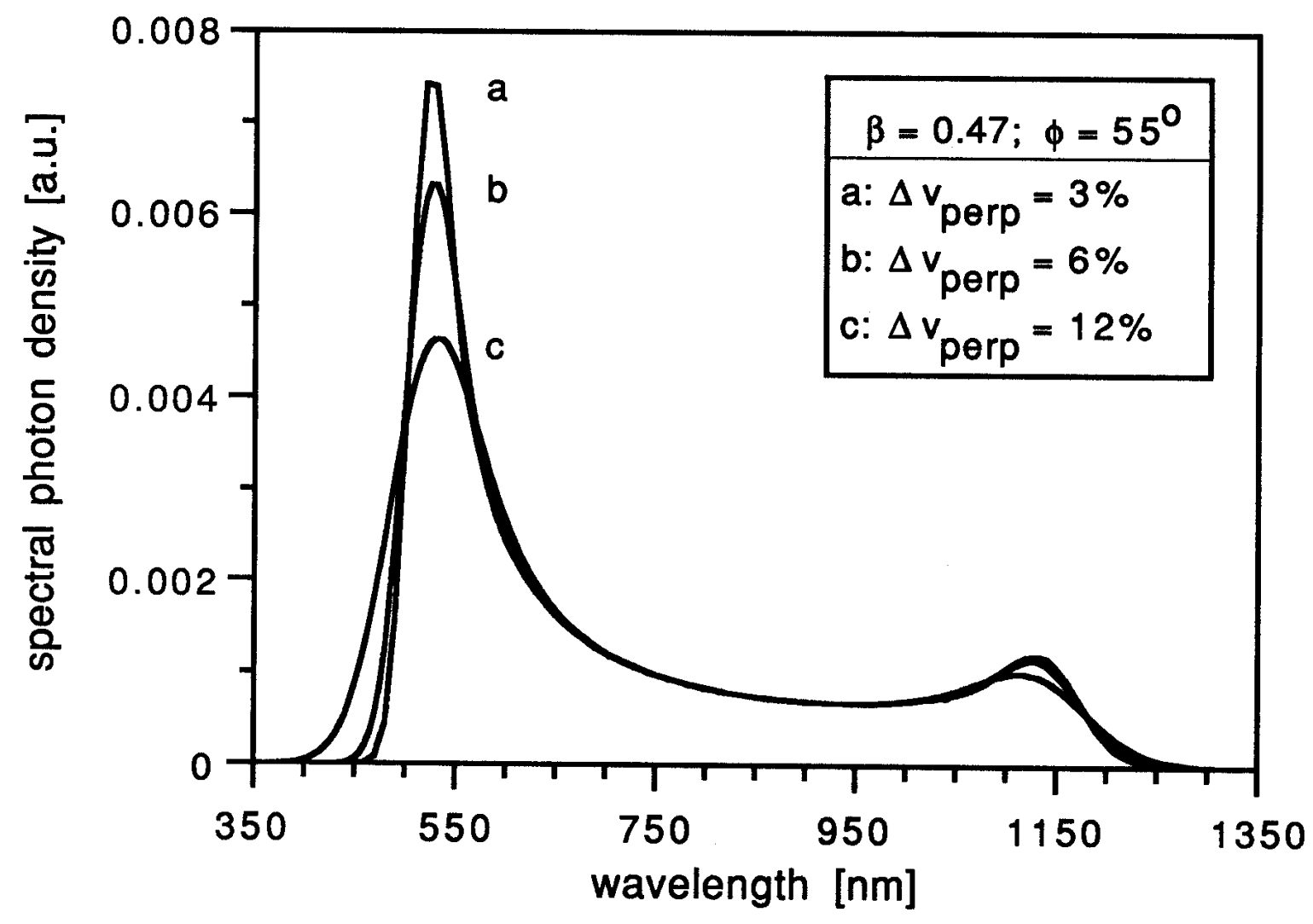

Fig, 8 (a) 


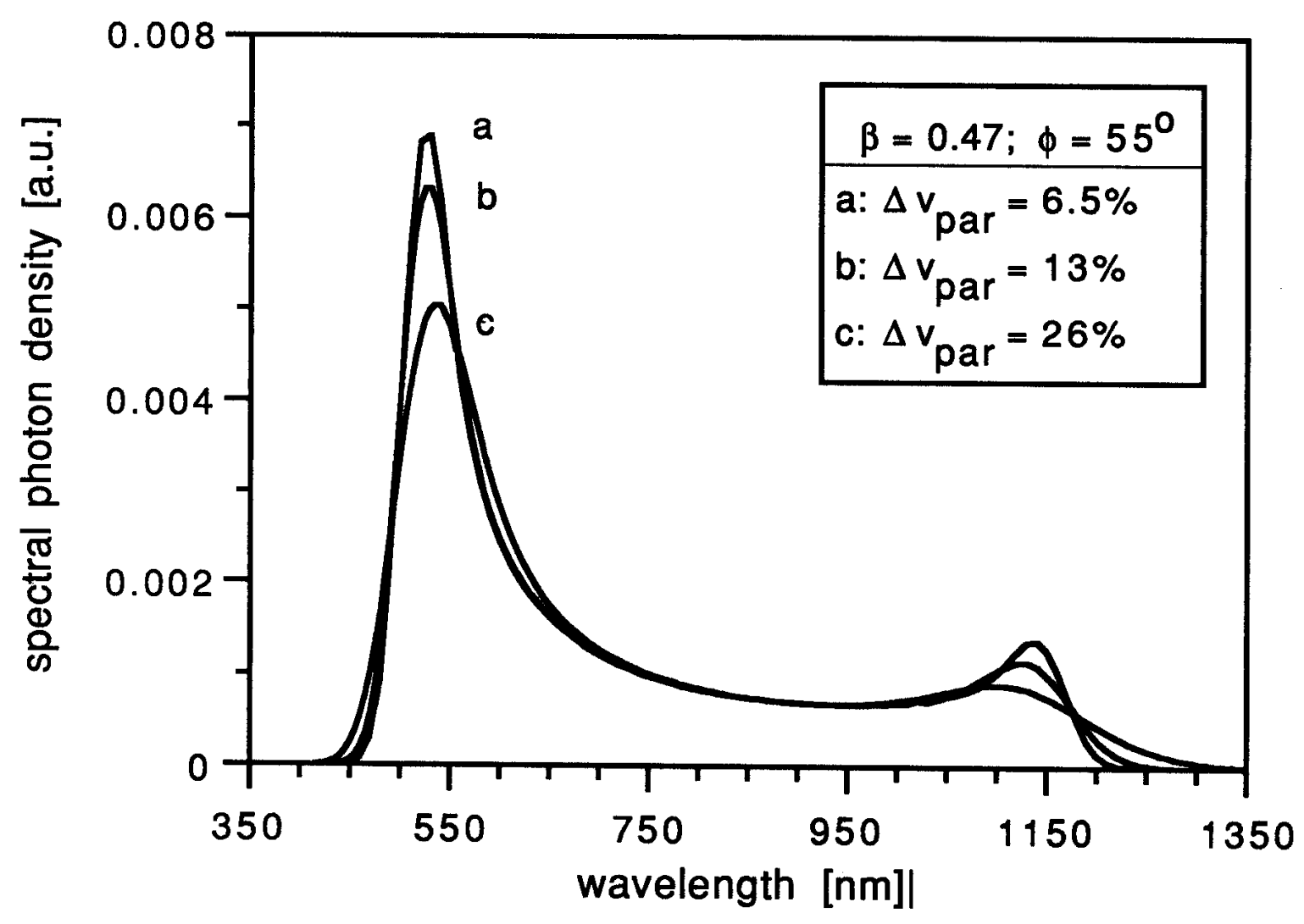

Fig, 3(b) 


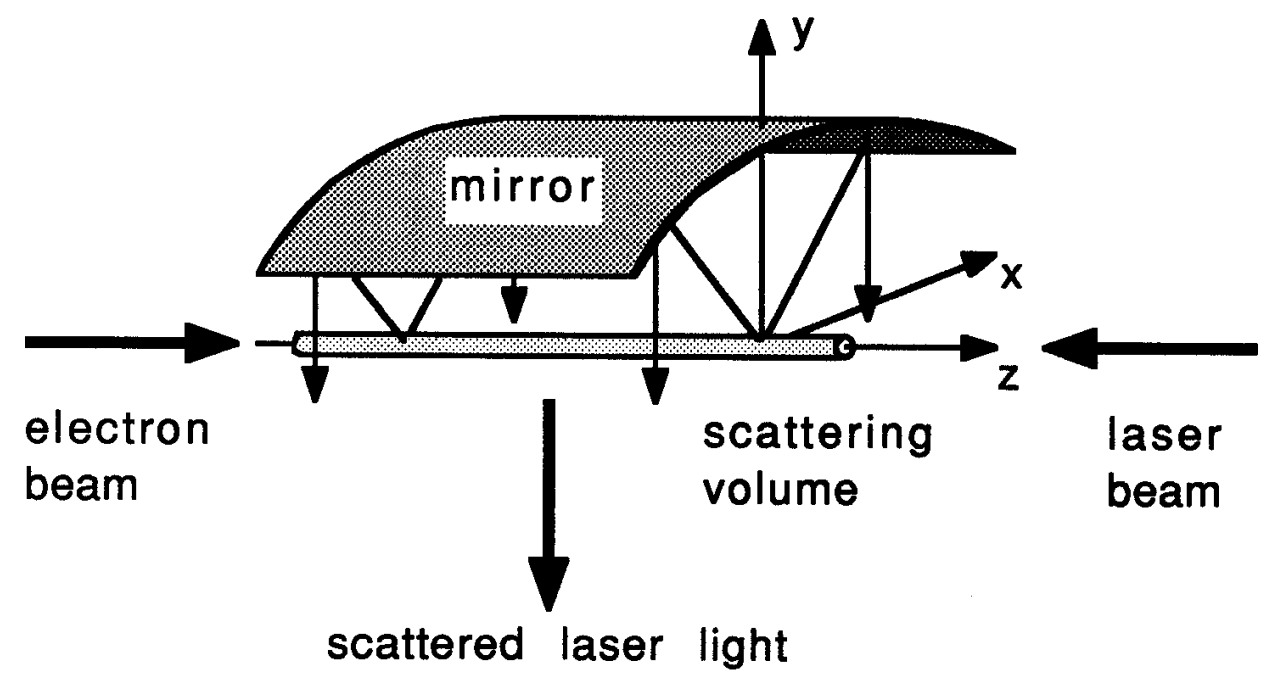

Fig, 9 

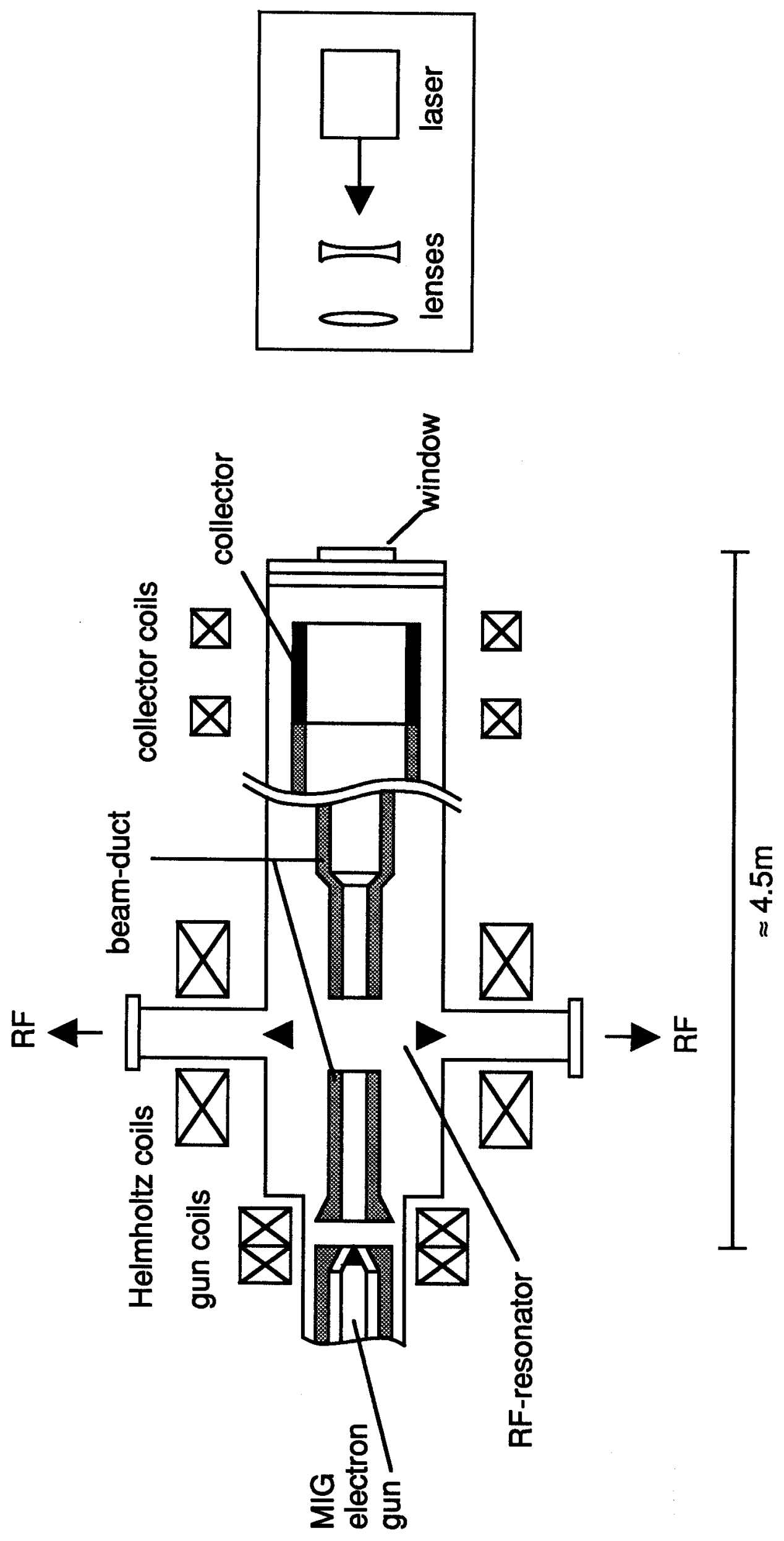

Fig, 10 


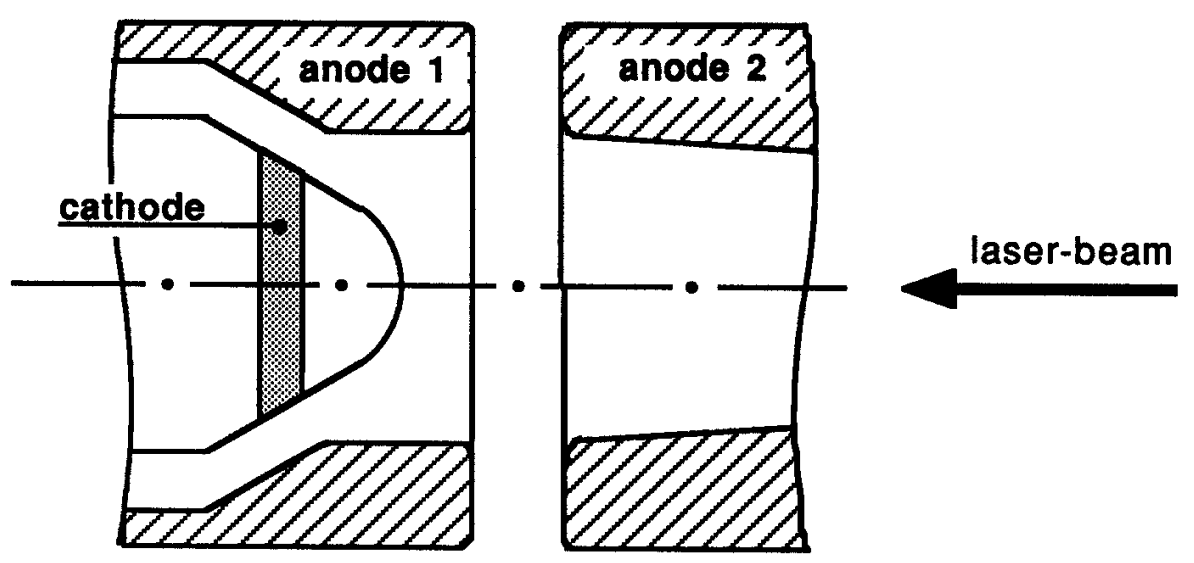

Fig, 11 